\title{
AIP1: a new player in TNF signaling
}

\author{
M. Eugenia Guicciardi and Gregory J. Gores
}

Division of Gastroenterology and Hepatology, Mayo Medical School, Clinic, and Foundation, Rochester, Minnesota, USA

Apoptosis signal-regulating kinase 1 (ASK1) is an upstream activator of JNK and p38 MAPK signaling cascades. Evidence now shows (see the related article beginning on page 1933) that the ASK1-interacting protein, AIP1, plays an important role in TNF- $\alpha$-induced ASK1 activation by facilitating dissociation from its inhibitor.

J. Clin. Invest. 111:1813-1815 (2003). doi:10.1172/JCI200318911.

The signaling system generated by the binding of TNF to its cognate TNFreceptor (TNF-R) can contain two distinct type I transmembrane receptors, TNF-R1 and TNF-R2, and three ligands, the membrane-bound TNF- $\alpha$, the soluble TNF- $\alpha$, and the soluble lymphocyte-derived cytokine lymphotoxin- $\alpha$ (LT- $\alpha)$ (1). Both receptors are ubiquitously expressed in cells and interact with both forms of TNF- $\alpha$, as well as with LT- $\alpha$. However, TNF-R1 is the most potent in inducing cytotoxic signals. TNF-R1 is a typical death receptor with a 60 - to 80 -amino acid cytoplasmic sequence known as the death domain. The death domain, which is missing from TNF-R2, typically enables death receptors to initiate cytotoxic signals. Because of the role of TNF- $\alpha$ in human diseases (2), TNF-R1 signaling mechanisms are of considerable biomedical interest.

\section{TNF-R1 signaling pathways}

The intracellular signals originating from TNF-R1 are extremely complex

\footnotetext{
Address correspondence to: Gregory J. Gores, Mayo Medical School, Clinic, and Foundation, 200 First Street SW, Rochester, Minnesota 55905, USA.

Phone: (507) 284-0686; Fax: (507) 284-0762; E-mail: gores.gregory@mayo.edu.

Conflict of interest: The authors have declared that no conflict of interest exists. Nonstandard abbreviations used: TNF receptor (TNF-R); lymphotoxin- $\alpha$ (LT- $\alpha$ ); Fasassociated protein with death domain (FADD); TNF receptor-associated factor-2 (TRAF-2); receptor-interacting protein (RIP); apoptosis signal-regulating kinase 1 (ASK1); ASK1interacting protein (AIP1); MAPK kinase (MAPKK); MAPK kinase kinase (MAPKKK); Ras GTPase-activating protein (Ras-GAP).
}

and can lead to multiple, even opposite, cell responses including cell proliferation, inflammation, or cell death. Survival signals likely prevail over death signals under normal circumstances, given the resistance of most cells to TNF- $\alpha$ induced toxicity. However, these cells can often be sensitized to TNF- $\alpha$-mediated apoptosis by blocking protein or RNA synthesis, which suggests that neosynthesis of protein is required to suppress the apoptotic stimulus. The expression of these antiapoptotic proteins is largely controlled by the activity of the transcription factor NF- $\kappa \mathrm{B}$, as inhibition of NF- $\mathrm{KB}$ also sensitizes cells to TNF- $\alpha$-induced cell death (3).

Binding of TNF-R1 to TNF- $\alpha$ results in conformational changes in the receptor's intracellular domain, resulting in rapid recruitment of several cytoplasmic death domain-containing adapter proteins via homophilic interaction with the death domain of the receptor (1). The first adaptor recruited to the clustered receptor is the TNF-R-associated protein with death domain, which functions as a docking protein for several signaling molecules, such as Fas-associated protein with death domain (FADD), TNF-R-associated factor-2 (TRAF-2), and receptor-interacting protein (RIP). Recruitment of FADD to the receptor promotes apoptosis by activation of caspase- 8 and, possibly, caspase-10 (4). RIP associates with TRAF- 2 to generate two distinct pathways (5). The first one signals through the activation of NF- $\mathrm{KB}-$ inducing kinase, NIK, and the catalytic IкB kinase complex, leading to phosphorylation of the NF- $\kappa \mathrm{B}$ inhibitory protein IкB- $\alpha$ and translocation of NF- $\kappa$ B to the nucleus. The second pathway involves the MAPK cascade, and culminates in the activation of JNK/ stress-activated protein kinase and p38 kinase. Both JNK and p38 play an important role in inflammatory responses. JNK has been described to phosphorylate and activate a number of transcription factors, including c-Jun, activating-transcription factor 2 and activator protein-1 (AP-1), which drive the expression of many proinflammatory molecules, including E-selectin, RANTES, IL-12, IL-6, and IL-8 (6). Activation of p38 is essential for production of proinflammatory cytokines such as IL- $1 \beta$, TNF- $\alpha$, and IL- 6 , and for induction and expression of inflammatory-related enzymes such as COX-2 and $\operatorname{iNOS}(7)$.

MAPKs are activated by a well-recognized mechanism of dual phosphorylation mediated by one of the MAP kinase kinases (MAPKKs), which, in turn, are activated through phosphorylation by MAP kinase kinase kinases (MAPKKKs) (Figure 1a). Previous studies have shown that TNF- $\alpha$-induced activation of JNK and p38 is mediated by the recruitment of the adaptor TRAF- 2 and subsequent activation of the MAPKKK apoptosis signal-regulating kinase 1 (ASK1) (8). ASK1, a 170-kDa Ser/Thr kinase, activates the JNK and p38 MAPK signaling cascades in response to proinflammatory cytokines and oxidative stress. Overexpression of ASK1 in epithelial cells induces apoptosis, whereas a kinaseinactive mutant of ASK1 protects the cells from TNF-induced apoptosis, suggesting that ASK1 is involved in the TNF apoptotic signaling pathway (9). Functionally, ASK1 is composed of an inhibitory $\mathrm{NH}_{2}$-terminal domain, an internal kinase domain, and a $\mathrm{COOH}$ terminal regulatory domain. The $\mathrm{COOH}$-terminal domain of ASK1 binds to the TRAF domain of TRAF-2 and TRAF-6 (8). The kinase domain is required for cytokine-induced JNK activation and cell death $(9,10)$. The $\mathrm{NH}_{2-}$ terminus contains an inhibitory domain that interacts with several cellular proteins and prevents ASK1 activation $(11,12)$. Although 14-3-3, a family of dimeric phosphoserine-binding molecules, binds to ASK1 specifically via 


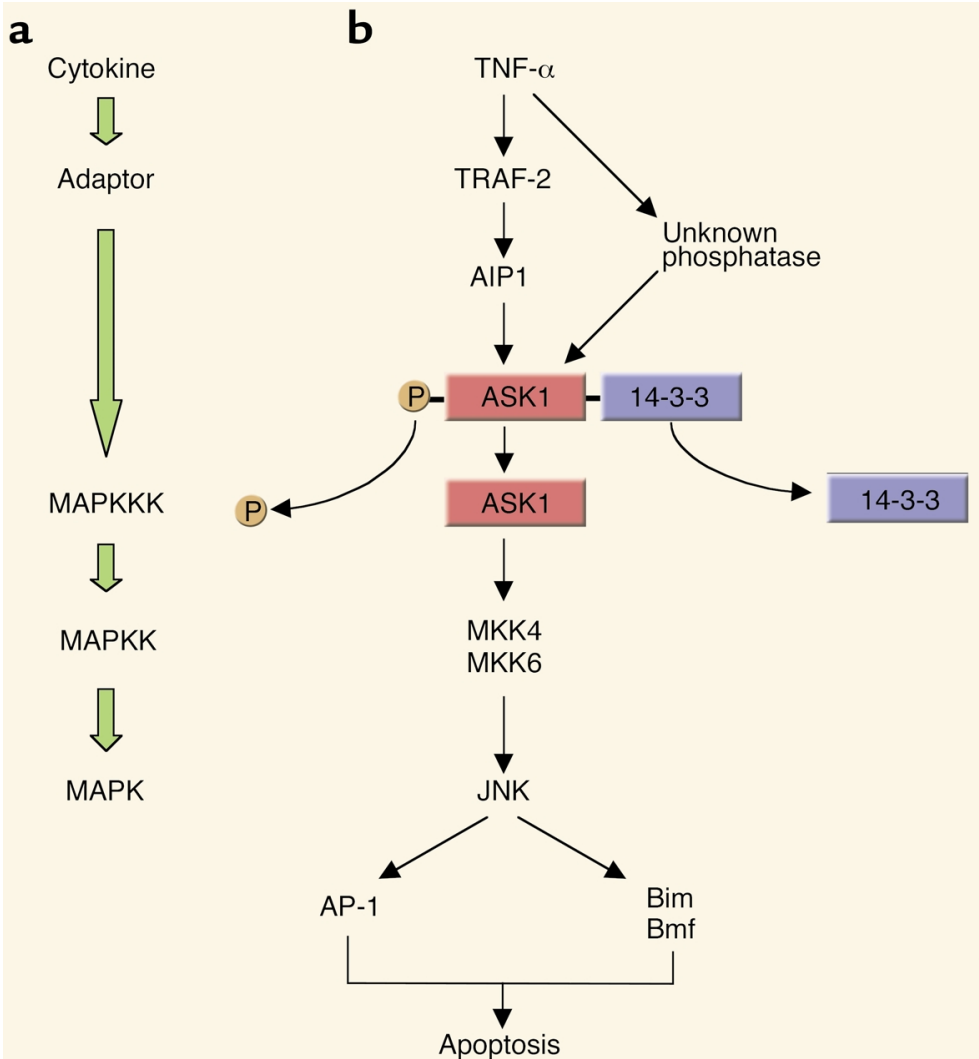

Figure 1

(a) Generic schema for the cytokine/adaptor/MAPK signaling paradigm. Cytokines, such as TNF- $\alpha$, trigger oligomerization of their enzymatically inactive cognate receptors. The conformational change in the receptor complex following ligand binding facilitates recruitment of adaptor proteins. These adaptor proteins within the receptor complex interact directly or indirectly with upstream kinases (MAPKKK) permitting their activation with subsequent phosphorylation and activation of downstream kinases (MAPKK/MAPK). (b) Model of TNF- $\alpha$ signaling pathway through TRAF2/ASK1 employing the cytokine/adaptor/MAPK paradigm. Triggering ofTNF-R1 leads to recruitment of the adaptor TRAF2, and concomitant activation of an unknown phosphatase via a mechanism yet to be identified. TRAF2 mediates the association of ASK1 with the newly identified Ras-GAP AIP1, which facilitates the release of ASK1 from its endogenous inhibitor 14-3-3. Disruption of the ASK1/14-3-3 complex and de-phosphorylation of ASK1 by the unknown phosphatase result in the activation of ASK1. ASK1, in turn, activates JNK via an MKK4- or MKK6-dependent pathway. Activation of JNK positively regulates the apoptotic machinery by transcription-dependent (i.e., activation of the transcription factor AP-1) and transcription-independent (i.e., phosphorylation and activation of proapototic $\mathrm{Bcl}-2$ proteins Bim and $\mathrm{Bmf}$ ) mechanisms. $\mathrm{P}$, phosphate.

Ser-967 in the COOH-terminal domain of ASK1, and inhibits ASK1-induced apoptosis (13), the precise role of 14-3-3 in regulating TNF-induced ASK1 activation has not been determined.

\section{Regulation of ASK-1}

In this issue of the JCI, Zhang and coworkers have described a new RasGTPase-activating protein (Ras-GAP), ASK1-interacting protein-1 (AIP1), which promotes TNF-induced activation of ASK1 by facilitating the dissociation of ASK1 from its endogenous as a chaperone, recruiting the phosphatase to ASK1. Supporting this interpretation, their data show that the binding site of AIP1 on ASK1 lies in a sequence surrounding the 14-3-3 binding site, and that AIP1, unlike 14-3-3, has higher affinity for the de-phosphorylated form of ASK1 at Ser-967. However, the nature of this unknown phosphatase and the temporal sequence of the events leading to the dissociation of the ASK1/14-3-3 complex remain to be elucidated. In fact, the identification, localization, and function of this phosphatase will be necessary to confirm and establish the proposed model. If proved, this model of ASK1 de-phosphorylation as an activation step for a MAPKKK would be unique.

The present paper from Zhang and coworkers (14) represents a significant scientific contribution by identifying the missing direct target of TRAF- 2 in the JNK pathway, AIP1. Regulation of ASK1 by AIP1 is likely very important in TNF- $\alpha$-mediated cytotoxic signaling as ASK1 is upstream of JNK. Activation of JNK can lead to apoptosis by transcriptionally-dependent and independent processes. JNK activation of the transcription factor AP-1 has been implicated in various models of cytotoxicity (16). Moreover, in a recent study, Lei and Davis describe a transcriptionallyindependent mechanism for JNK associated apoptosis by demonstrating that JNK directly phosphorylates the proapoptotic $\mathrm{BH} 3$-only proteins Bim and Bmf promoting mitochondrial dysfunction with cytochrome $c$ release (17). The role of ASK1 in initiating this cascade requires further clarification.

This paper also highlights the role of 14-3-3 in regulating apoptosis. Phosphorylation of Bad, another proapoptotic BH3-only member of the Bcl-2 family, also promotes its recruitment and sequestration by 14-3-3 proteins $(18,19)$. This mechanism of inactivation of Bad is highly reminiscent of the one operated by 14-3-3 on ASK1, suggesting that one of the functions of 14-3-3 proteins is to support cell survival by inhibiting the activity of proapoptotic proteins.

In conclusion, the identification of AIP1 by Zhang and co-workers (14) provides a further detail of the TNF-R1 MAPK cascade and helps to unravel the 
molecular bases of the TNF signaling. Given its important contribution to TNF-induced JNK activation, AIP1 may represent a suitable target for possible therapeutic applications in human diseases characterized by increased TNF- $\alpha$-mediated apoptosis.

\section{Acknowledgments}

This work was supported by grants from the NIH (DK 63947) and the Mayo Foundation.

1. Wallach, D., et al. 1999. Tumor necrosis factor receptor and Fas signaling mechanisms. Ann. Rev. Immunol. 17:331-367.

2. McDermott, M.F. 2001. TNF and TNFR biology in health and disease. Cell. Mol. Biol. 47:619-635.

3. Wang, C.Y., Mayo, M.W., Korneluk, R.G., Goeddel, D.V., and Baldwin, A.S. 1998. NF-кB antiapoptosis: induction of TRAF 1 and TRAF 2 and c-IAP1 and c-IAP2 to suppress caspase- 8 activation. Science. 281:1680-1683.

4. Kischkel, F.C., et al. 2001. Death receptor recruitment of endogenous caspase- 10 and apoptosis initiation in the absence of caspase-8. J. Biol. Chem. 276:46639-46646.

5. Wajant, H., and Scheurich, P. 2001. Tumor necrosis factor receptor-associated factor (TRAF) 2 and its role in TNF signaling. Int. J. Biochem. Cell. Biol. 33:19-32.

6. Ip, Y.T., and Davis, R.J. 1998. Signal transduction by the c-jun $\mathrm{N}$-terminal kinase (JNK): from inflammation to development. Curr. Opin. Cell Biol. 10:205-219.

7. Ono, K., and Han, J. 2000. The p38 signal transduction pathway: activation and function. Cell Signal. 12:1-13.

8. Nishitoh, H., et al. 1998. ASK1 is essential for JNK/SAPK activation by TRAF2. Mol. Cell. 2:389-395.

9. Chang, H.Y., Nishitoh, H., Yang, X., Ichijo, H., and Baltimore, D. 1998. Activation of apoptosis signal-regulating kinase 1 (ASK1) by the adapter protein Daxx. Science. 281:1860-1863.

10. Ichijo, H., et al. 1997. Induction of apoptosis by ASK1, a mammalian MAPKKK that activates SAPK/JNK and p38 signaling pathways. Science. 275:90-94.

11. Liu, H., Nishitoh, H., Ichijo, H., and Kyriakis, J.M. 2000. Activation of apoptosis signal-regulating kinase 1 (ASK1) by tumor necrosis factor receptor-associated factor 2 requires prior dissociation of the ASK1 inhibitor thioredoxin. Mol. Cell. Biol. 20:2198-2208

12. Saitoh, M., et al. 1998. Mammalian thioredoxin is a direct inhibitor of apoptosis signal- regulat ing kinase (ASK) 1. EMBOJ. 17:2596-2606.

13. Zhang, L., Chen, J., and Fu, H. 1999. Suppression of apoptosis signal-regulating kinase 1 -induced cell death by 14-3-3 proteins. Proc. Natl. Acad. Sci. U. S. A. 96:8511-8515.

14. Zhang, R., et al. 2003. AIP1 mediates TNF- $\alpha-$ induced ASK1 activation by facilitating dissociation of ASK1 from its inhibitor 14-3-3 J. Clin. Invest. 111:1933-1943. doi:10.1172/ JCI200317790.

15. Liu, Y., Yin, G., Surapisitchat, J., Berk, B.C., and Min, W. 2001. Laminar flow inhibits TNFinduced ASK1 activation by preventing dissociation of ASK1 from its inhibitor 14-3-3. J. Clin. Invest. 107:917-923.

16. Jones, B.E., and Czaja, M.J. 1998. Mechanisms of hepatic toxicity. III. Intracellular signaling in response to toxic liver injury. Am. J. Physiol. Gastrointest. Liver Physiol. 275:G874-G878.

17. Lei, K., and Davis, R.J. 2003. JNK phosphorylation of Bim-related members of the Bcl-2 family induces Bax-dependent apoptosis. Proc. Natl. Acad. Sci. U. S. A. 100:2432-2437.

18. Zha, J., Harada, H., Yang, E., Jockel, J., and Korsmeyer, S.J. 1996. Serine phosphorylation of death agonist BAD in response to survival factor results in binding to 14-3-3 not Bcl- $\mathrm{X}_{\mathrm{L}}$. Cell. 87:619-628.

19. Datta, S.R., et al. 2000. 14-3-3 proteins and survival kinases cooperate to inactivate $\mathrm{BAD}$ by $\mathrm{BH} 3$ domain phosphorylation. Mol. Cell. 6:41-51.

\title{
Arginase: marker, effector, or candidate gene for asthma?
}

\section{Donata Vercelli}

Arizona Respiratory Center, College of Medicine, University of Arizona, Tucson, Arizona, USA

\begin{abstract}
Microarray analysis of the expression profiles of lung tissue in two murine models of asthma revealed high levels of arginase I and arginase II activity, in association with IL-4 and IL-13 overexpression (see the related article beginning on page 1863), suggesting that arginine pathways are critical in the pathogenesis of asthma.
\end{abstract}

J. Clin. Invest. 111:1815-1817 (2003). doi:10.1172/JCI200318908.

Despite intense research efforts, asthma remains a major medical and scientific challenge. Prevalence of this disease increased 75\% between 1980 and 1998 . Although this rate may now be stabilizing, the 2001 National Health Interview Survey estimated that $6.9 \%$ of adults, and $8.9 \%$ of children under the age of 18 in the United States, suffered from

Address correspondence to: Donata Vercelli, College of Medicine, University of Arizona, 1501 North Campbell Avenue, Suite 2349, Tucson, Arizona 85724-5030, USA. Phone: (520) 626-6387; Fax: (520) 626-6970; E-mail: donata@resp-sci.arizona.edu.

Conflict of interest: The author has declared that no conflict of interest exists.

Nonstandard abbreviations used: protein inhibitor of activated STAT1 (PIAS1). asthma (1). The reasons why asthma prevalence has been on the rise for so long remains a matter of intense speculation. The pathogenetic mechanisms of the disease, and the contributing genetic factors, also remain elusive. This state of affairs probably reflects the inherent complexity of the disease, and the difficulty associated with stringently defining asthmatic phenotypes so that homogenous subject groups can be identified for mechanistic studies.

\section{Microarrays: a powerful tool to dissect asthma}

An aggressive approach to the identification of new asthma genes is discussed in this issue of the JCI by Zimmermann and collaborators (2), who determined transcript expression profiles in lung tissue from mice with an asthma-like phenotype induced by sensitization with OVA or Aspergillus fumigatus. The recognized strength of microarray experiments lies in their ability to address an issue globally, and highlight the unexpected. The results of this study are no exception. An important quantitative finding was that $6.5 \%$ of the 12,422 genes analyzed showed a greater than twofold change in expression in challenged mice. These data show that, although asthma remains confined to the lung, the mechanistic dysregulation underlying the disease - whatever that may be - mobilizes a vast genetic program. Even more importantly, among the 496 and 527 genes identified in the OVA and Aspergillus models, respectively, only 291 were common to both. Since all mice had the same genetic background, this pattern is likely to result from differences in pathogenetic mechanisms, possibly related to the nature of the allergen and/or the immunization route. Such data should provide molecular epidemiologists and clinicians interested in asthma with spicy food for thought.

\section{Enter arginine and its pathways}

Intriguing findings also came from the qualitative analysis of lung transcript 